\title{
Leveraging Card-based Collaborative Activities as Culturally Situated Design Tools
}

\author{
D. Scott McCrickard ${ }^{1}$, DeMarcus Townsend ${ }^{1}$, \\ Woodrow W. Winchester, III $^{2}$, and Tiffany Barnes ${ }^{3}$ \\ ${ }^{1}$ Virginia Tech, Department of Computer Science, 2202 Kraft Drive, \\ Blacksburg, VA 24060, United States \\ ${ }^{2}$ Virginia Tech, Grado Department of Industrial and Systems Engineering, 250 Durham \\ Hall, Blacksburg, VA 24061, United States \\ ${ }^{3}$ University of North Carolina, Charlotte, Department of Computer Science, \\ Charlotte, NC, 28223, United States \\ \{mccricks,dm2town,wwwinche\}@vt.edu, tiffany.barnes@uncc.edu
}

\begin{abstract}
This paper describes two examples of virtual card games serving as Culturally Situated Design Tools (CSDTs) for young people. CSDTs have promise in helping people to learn by connecting principles from computing with aspects of their heritage or gender. The development and deployment of card games on two cutting-edge platforms (mobile devices and multitouch tables) revealed novel ways to display information to users and important lessons for deploying them to young people.
\end{abstract}

Keywords: culturally situated design tools, games, education, mobile, multitouch.

\section{Introduction}

Culturally Situated Design Tools (CSDTs) seek to help people to learn by connecting principles from computing and technology with aspects of their heritage valued by members who identify with a demographic or culture. These aspects can include clothing that they wear, food that they eat, crafts that they undertake, activities that they do, people with whom they identify, or other cultural aspects that are particularly valued or related to the demographic. The theory behind this approach is that the unique aspects will provide context and meaning to underrepresented groups, thus overcoming the often unintentional biases of the majority group in choosing teaching tools and techniques.

This work seeks to leverage collaborative card games to connect with particular focus on two groups: women and African-Americans. Both groups historically play card games at a disproportionate rate, and both put unique value on the importance of collaboration through games. In modern times, evidence shows rapid adoption of mobile and non-traditional technology by women and African-Americans due to the availability of collaborative activities and the ability to strengthen interpersonal relationships. This work seeks to use novel emerging technologies to leverage the 
cultural appeal of card-based games to attract young people (K-12 students, in particular middle and high school students from underrepresented groups) to computing and technology fields. This paper describes two design and evaluation examples by the authors and their collaborators that embody card game motifs: HealthAttack for the iPhone and METapp for multitouch surfaces. Drawing from the experiences with these games, the paper concludes with a roadmap for future cardbased application development and testing.

\section{Background and Approach}

Culturally-Situated Design Tools (CSDTs) are activities based on math and computing knowledge; examples include the use of African American cornrow hairstyles to explore computation [1] and the use of Native American beadwork to show scanning algorithms [2]. CSDTs are used to teach people about difficult computational concepts, with the expectation that people from the represented groups (i.e., African Americans or Native Americans) will be motivated to learn because of the cultural connection.

This paper explores the use of card games as a CSDT. Unlike many computing activities, members of underrepresented groups-especially women, African Americans, and Hispanics) play certain games at equal or greater rates than white males [3]. Among those are card games, which often have cultural roots in underrepresented groups. Card games provide an experience for users that is both engaging and informative. Games have been leveraged previously to reach out to both adults and children, e.g., [4]. This game reinforces what is learned in school about the USDA food pyramid. As it was the developers' collective belief that responsiveness to cultural norms is of importance in the usefulness of a health and wellness application, the game leverages cultural norms and values central to the African-American community such as "role-modeling of behaviors".

\section{Example 1: Health Attack}

One common category of card games are memory-based games, in which participants seek to match pairs of cards from a large set of unordered pairs that are initially face down in a grid. Our efforts sought to implement such a game on a mobile device, with a focus on ethnic foods to increase appeal to underrepresented populations. Implementation on a mobile device also allowed us to extend the capabilities of the game to provide information about the foods, specifically nutrition information and ways to cook the food in a healthy manner. We called the game "Health Attack" and targeted it for African American youth. This section of the paper describes Health Attack as a tool for culturally situated design and understanding; for a more complete description of the implementation, use, and evaluation, see [5].

Health Attack is one of a large collection of applications for "smart" phones that are or will soon be distributed through the marketplace environments for the Apple iPhone and Google Android platforms. Smart phones are particularly relevant for 
many minority populations as they are often their primary Internet access platform [6]. Researchers in our labs have distribution experience through these venues as registered developers, with some applications receiving hundreds of downloads in as little as two months.

A team of designers was assembled and charged with creating an engaging interface to address health and wellness concerns among African Americans. Five of the seven people on this team were African American, providing connections and empathy with the target population with potential for improved interfaces [7]. The design team sought to target a younger family demographic-children ages 7-11expecting that behavior would be easier to influence and that the lessons would be reinforced in schools and other community groups.

With the African American culture in mind, the developers designed the interactive game for African-American children to provide awareness of nutritional information of commonly-eaten food. Based on the literature, field studies, and relevant cultural elements central to the African-American community (drawing heavily from their own experiences as youth and as older siblings), the developers identified symbols of black culture in our surrounding communities by going around the local community and to take pictures and notes about African American culture-quickly centering on issues of food selection and preparation. After finding important symbols in the community, the design team discussed how to convert that same tradition and important characteristics into the application targeted for African-American children.

The Health Attack game augments the traditional memory matching game by asking players to match food items that are placed in their respective places on the USDA food pyramid (see Figure 1). While users are playing the matching game, they are also given nutrition and health-related facts about each match. This feedback balances fun and learning, to attract users and to give them a reason to continue learning more about health and nutrition. By showing foods popular among AfricanAmericans, Health Attack provides a sense of ownership for the target users. Also included on each card are quotes from African-American role models to help make the game feel more authentic and accessible to the African American community.
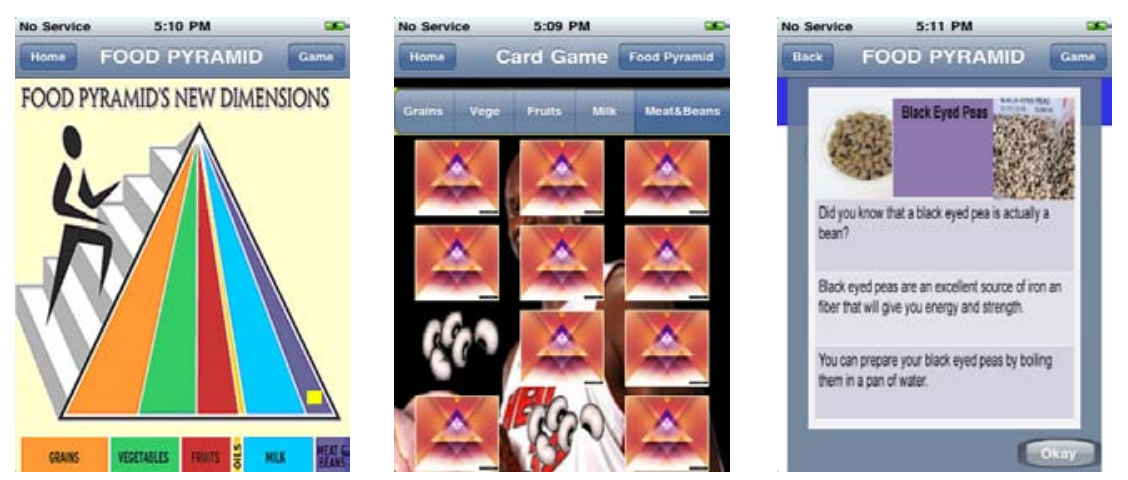

Fig. 1. Health Attack screens: Pyramid view (left) adapted from the USDA food pyramid; Game View (center) showing a match; Information View (right) with detailed information 
Evaluation of Health Attack was conducted through a demo and questionnaire. The feedback questionnaire was given out to sixteen participants-primarily K-12 teachers, but also some college teachers and senior students. The participants viewed information on a poster, talked with developers and project leads, and experienced hands-on use of the application at a highly interactive poster session. The participants felt that the application would be helpful to raise nutritional awareness for children, specifically African-American children. In particular, there were positive feelings about the different role models used in the backgrounds throughout the game-though two of the participants did not answer this question, perhaps because they did not notice the role models. Since many of the participants were not African American or did not understand the African American culture, they may not have felt comfortable addressing some of the issues; future study will focus within the demographic.

\section{Example 2: MET apps for multitouch tables}

The second example is the multitouch educational table application (MET) for multitouch tabletop computing technologies that provides a platform for collaborative and competitive card games. We designed this in-house technology to support multiperson, multi-handed interaction. A signature series of games asks participants to match cards with the same picture (in simple mode for younger players) or to match inventors and their inventions (for more advanced play). Some card sets focus on inventions by female and African American inventors, in an effort to connect better with those demographics. See [8] for a report of the design and assessment of MET.

Our team has explored the utility of multitouch tables through both field interventions and lab-based studies. The field interventions were highly informative; most notably in highlighting issues with the appeal to girls. Often the competitive nature of some of the games seemed to dissuade girls from playing the games. They tended to be more hesitant, seeking to learn the rules and to wait for a demo from a facilitator before starting the game. A lab-based usability study comparing METapp to a comparable computer desktop application showed that performance is similar across the different platforms, but participants enjoy the multitouch table game more than the traditional desktop game. While there were no differences in performance by minority groups, prior research suggests that long-term learning benefits may exist (see [7]).

Our planned MET game seem likely to address some of the barriers due to the competitiveness. As an example, our ongoing work seeks to create a collaborative game (more an activity) to provide a face-to-face Facebook-based experience in which multiple participants indicate their preference for music, books, and other topics that, when aggregated and displayed, can serve as the basis for conversations, collaborations, and friendships. The simple elegance of this type of application (on a cutting-edge platform) will hopefully serve to demystify computing and technology at an important age for recruitment to the field. Continued testing with K-12 groups will further reveal the effectiveness of these methods as a tool for encouraging technology use and as a method for encouraging collaboration and communication. 


\section{Conclusions and Future Work}

Our experiences with these two example games suggest the following conclusions and directions for future work. First, there is promise in card-based games and activities, as students who were unfamiliar with these technologies tended to learn them quickly and embrace them readily. Second, we found that card-based games and activities benefit from highly collaborative (and sometimes competitive) aspects to them. Third, the appeal of cutting-edge technology serves as a recruiting tool, particularly in our involvement with middle and high school students, including groups that traditionally attract girls and minority students. We are inspired by and encouraged by the many other efforts in designing for minority populations, particularly the work of Grimes and Grinter (e.g., [9]). We feel that the true value of this work will take place with the technologies in the hands of people who can benefit from them.

\section{Acknowledgements}

Thanks goes to the NSF for grants IIS-0851774 and CNS-0940358 that supported this work. The opinions in this paper are ours and not necessarily shared by the NSF. Also, thanks to the many people who helped build and use our methods and software.

\section{References}

1. Eglash, R. and Bennett, A. Teaching with hidden capital: Agency in children's computational explorations of cornrow hairstyles. Children, Youth and Envinroments 19 (1), 58-73 (2009)

2. Eglash, R. Ethnocomputing with Native American Design. Information Technology and Indigenous People, chapter 29, 210-219 (2007)

3. Markin, G.A. Game Demographics—She's Young, She’s Practiced, She’s Good. Alien Babel Tech, http://alienbabeltech.com/main/?p=20058, downloaded 1/12/2011

4. Consolvo, S., McDonald, D. W., and Landay, J. A. Theory-driven design strategies for technologies that support behavior change in everyday life. In: Proceedings of the ACM Conference on Human Factors in Computing Systems (CHI 2009), 405-414, 2009

5. Hill, D., Blunt, J. Pugh, T., Monk, M., Kim, J.-S., McCrickard, D.S., Winchester, W.W., Estabrooks, P., and Doswell, F. Mobile technologies for promoting health and wellness among African American youth. In Proceedings of HCII (2011)

6. Smith, A. Mobile Access. Technical report, Pew Internet \& American Life Project (2010)

7. Winchester, W. W., McCrickard, D. S., and Doswell, F. Towards culturally empowered design: Addressing African-American health disparities through notification interfaces. Workshop paper in CHI Wellness Information Workshop, 4 pages (2010)

8. George, J., de Araujo, E., Dorsey, D., McCrickard, D.S., and Wilson, G. Multitouch tables for collaborative object-based learning. In: Proceedings of HCII (2011)

9. Grimes, A. and Grinter, R. E. Designing persuasion: Health technology for low-income African American communities. In: Proceedings of Persuasive Technology, 24-35 (2007) 